\title{
AN $m$-ORTHOCOMPLETE ORTHOMODULAR LATTICE IS $m$-COMPLETE
}

\author{
SAMUEL S. HOLLAND, JR.
}

ABSTRACT. We call an orthomodular lattice $\mathscr{L} m$-orthocomplete for an infinite cardinal $m$ if every orthogonal family of $\leqq m$ elements from $\mathscr{L}$ has a join in $\mathscr{L}$, and we call $\mathfrak{L} m$-complete if every family, orthogonal or not, of $\leqq m$ elements from $\mathscr{L}$ has a join in $\mathscr{L}$. We prove that an $m$-orthocomplete orthomodular lattice is $m$ complete. Since a Boolean algebra is a distributive orthomodular lattice, we obtain as a special case the Smith-Tarski theorem: An $m$-orthocomplete Boolean algebra is $m$-complete.

We refer the reader to [1] for the elementary theory and basic nomenclature of orthomodular lattices, mentioning specifically here only these notational conventions: we write $a-b$ for $a \wedge b^{\perp}$ when $b \leqq a$, and write $\bigoplus a_{\alpha}$ for $\bigvee a_{\alpha}$ when $\alpha \neq \beta \Rightarrow a_{\alpha} \perp a_{\beta}$.

Lemma. Let $\&$ be an m-orthocomplete orthomodular lattice, $\sigma$ an ordinal number satisfying $\operatorname{card}(\sigma) \leqq m$, and $\left(y_{\alpha} ; \alpha<\sigma\right)$ a family of elements from L satisfying

(i) $y_{0}=0$,

(ii) $\alpha \leqq \beta<\sigma \Rightarrow y_{\alpha} \leqq y_{\beta}$ (increasing),

(iii) $\beta$ a limit ordinal $<\sigma \Rightarrow \bigvee\left(y_{\alpha} ; \alpha<\beta\right)$ exists and $=y_{\beta}$ (continuous from the left).

Then for every ordinal $\beta$ satisfying $2 \leqq \beta<\sigma$ we have

$$
\mathrm{V}\left(y_{\alpha} ; \alpha<\beta\right)=\bigoplus\left(y_{\rho+1}-y_{\rho} ; \rho+1<\beta\right) .
$$

Proof of the Lemma. Both joins displayed in the assertion of the lemma exist, the orthogonal join by $m$-orthocompleteness, and the other by assumption (iii). (Assumption (iii) covers the case when $\beta$ is a limit ordinal; if $\beta$ is not a limit ordinal, then obviously $\mathrm{V}\left(y_{\alpha} ; \alpha<\beta\right)=y_{\beta-1}$. $)$ If $\rho+1<\beta$, then $y_{\rho+1}-y_{\rho} \leqq y_{\rho+1} \leqq \mathrm{~V}\left(y_{\alpha} ; \alpha<\beta\right)$; hence

$$
\bigoplus\left(y_{\rho+1}-y_{\rho} ; \rho+1<\beta\right) \leqq \mathrm{V}\left(y_{\alpha} ; \alpha<\beta\right) .
$$

We need therefore prove only the statement $P(\beta): \mathrm{V}\left(y_{\alpha} ; \alpha<\beta\right)$ $\leqq \bigoplus\left(y_{\rho+1}-y_{\rho} ; \rho+1<\beta\right) . P(2)$ is the assertion $y_{1} \leqq y_{1}-y_{0}$ which is true because $y_{0}=0$. We use transfinite induction. Assume that $P(\gamma)$

Received by the editors June 30, 1969.

A MS Subject Classifications. Primary 0635, 0660.

Key Words and Phrases. Orthomodular lattices, Boolean algebras, $m$-orthocomplete, $m$-complete, Smith-Tarski theorem. 
is true for all $\gamma<\beta$. If $\beta$ is a limit ordinal, then for any $\alpha<\beta, \alpha+1<\beta$ and then, using the induction hypothesis,

$$
\begin{gathered}
y_{\alpha}=\mathrm{V}\left(y_{\sigma} ; \sigma \leqq \alpha\right)=\mathrm{V}\left(y_{\sigma} ; \sigma<\alpha+1\right) \\
\leqq \bigoplus\left(y_{\rho+1}-y_{\rho} ; \rho+1<\alpha+1\right) \leqq \bigoplus\left(y_{\rho+1}-y_{\rho} ; \rho+1<\beta\right) .
\end{gathered}
$$

Hence $\mathrm{V}\left(y_{\alpha} ; \alpha<\beta\right) \leqq \bigoplus\left(y_{\rho+1}-y_{\rho} ; \rho+1<\beta\right)$. If $\beta$ is not a limit ordinal, then $\mathrm{V}\left(y_{\alpha} ; \alpha<\beta\right)=\mathrm{V}\left(y_{\alpha} ; \alpha \leqq \beta-1\right)=y_{\beta-1}$. Now there are two possibilities: either $\beta-1$ is a limit ordinal or it is not. If $\beta-1$ is a limit ordinal, then by (iii) and the induction hypothesis,

$$
\begin{aligned}
y_{\beta-1} & =\bigvee\left(y_{\alpha} ; \alpha<\beta-1\right) \leqq \bigoplus\left(y_{\rho+1}-y_{\rho} ; \rho+1<\beta-1\right) \\
& \leqq \bigoplus\left(y_{\rho+1}-y_{\rho} ; \rho+1<\beta\right)
\end{aligned}
$$

and we are done. If $\beta-1$ is not a limit ordinal, then

$$
\begin{aligned}
\bigoplus\left(y_{\rho+1}-y_{\rho} ; \rho\right. & +1<\beta) \\
= & \oplus\left(y_{\rho+1}-y_{\rho} ; \rho+1 \leqq \beta-1\right) \\
& =\left(y_{\beta-1}-y_{\beta-2}\right) \oplus \oplus\left(y_{\rho+1}-y_{\rho} ; \rho+1<\beta-1\right) \\
& \geqq\left(y_{\beta-1}-y_{\beta-2}\right) \oplus \vee\left(y_{\alpha} ; \alpha<\beta-1\right) \\
& =\left(y_{\beta-1}-y_{\beta-2}\right) \oplus y_{\beta-2}=y_{\beta-1},
\end{aligned}
$$

which proves $P(\beta)$. (In the second to the last step we used the induction hypothesis.)

TheOREM. An m-orthocomplete orthomodular lattice is m-complete.

Proof. By induction. Let $\left(x_{\gamma} ; \gamma \in \Sigma\right)$ be a family of elements from $\mathscr{L}$ indexed by a set $\Sigma$ with $\operatorname{card}(\Sigma) \leqq m$, and assume that the join of any $\Sigma^{\prime}$-indexed family exists when $\operatorname{card}\left(\Sigma^{\prime}\right)<\operatorname{card}(\Sigma)$. Let $\sigma$ be the least ordinal corresponding to $\operatorname{card}(\Sigma)$. We can suppose that $\operatorname{card}(\Sigma)$ is infinite so that $\sigma$ is a limit ordinal, and we can suppose that we have replaced the set $\Sigma$ by the set $(\alpha ; \alpha<\sigma)$ so that we are dealing with an ordinal-indexed family $\left(x_{\alpha} ; \alpha<\sigma\right)$. By the induction assumption $y_{\alpha}=\mathrm{V}\left(x_{\rho}, \rho<\alpha\right)$ exists for every $\alpha<\sigma$. This family $\left(y_{\alpha} ; \alpha<\sigma\right)$ satisfies the conditions of the lemma, (i) and (ii) being obviously met, and (iii) being a consequence of the following direct computation for $\beta$ a limit ordinal $<\sigma: \bigvee\left(y_{\alpha} ; \alpha<\beta\right)=\mathrm{V}_{\alpha<\beta} \mathrm{V}\left(x_{\rho} ; \rho<\alpha\right)=\mathrm{V}\left(x_{\rho} ; \rho<\beta\right)$ $=y_{\beta}$.

The orthogonal join $z=\bigoplus\left(y_{\alpha+1}-y_{\alpha} ; \alpha+1<\sigma\right)$ exists by $m$-orthocompleteness; this element $z$ is the desired join, $\mathrm{V}\left(x_{\rho} ; \rho<\sigma\right)$.

First, note that if $z$ is in fact an upper bound of the set $\left(x_{\rho} ; \rho<\sigma\right)$, then, among all such upper bounds, it is certainly the least. For if $w \geqq x_{\rho}$ for all $\rho<\sigma$, then $w \geqq \bigvee\left(x_{\rho} ; \rho<\alpha+1\right)=y_{\alpha+1} \geqq y_{\alpha+1}-y_{\alpha}$ for all 
$\alpha+1<\sigma$ so $w \geqq z$. Hence it is enough to show that $z \geqq x_{\beta}$ for every $\beta<\sigma$.

If $\beta<\sigma$ then, $\sigma$ being a limit ordinal, we have $\beta+2<\sigma$, whence

$$
\begin{aligned}
x_{\beta} & \leqq \mathrm{V}\left(x_{\rho} ; \rho<\beta+1\right)=y_{\beta+1}=\mathrm{V}\left(y_{\alpha} ; \alpha \leqq \beta+1\right) \\
& =\mathrm{V}\left(y_{\alpha} ; \alpha<\beta+2\right)=\bigoplus\left(y_{\rho+1}-y_{\rho} ; \rho+1<\beta+2\right) \leqq z,
\end{aligned}
$$

where, in the second-to-the last step, we have used the lemma. That proves the theorem.

Call an orthomodular lattice $\mathbb{L}$ orthocomplete if it is $m$-orthocomplete for every $m$ (or for $m=\operatorname{card}(\&)$ which is enough).

Corollary 1. An orthocomplete orthomodular lattice is complete.

An orthomodular lattice $\&$ satisfies the " $m$-chain condition" (I am adapting this nomenclature from Sikorski [2, p. 72]) provided that any orthogonal family in $\&$ has $\leqq m$ nonzero elements.

COROllary 2. An m-orthocomplete orthomodular lattice satisfying the $m$-chain condition is complete.

For $m=\boldsymbol{\aleph}_{0}$, this was proved by Zierler [3, Lemmas 1.8 and 1.9].

Corollary 3 (Smith-Tarski; SEE $[2 ; \S 20.1]$ ). An m-orthocomplete Boolean algebra is $m$-complete.

Corollary 4 (TARSKI; SEE $[2 ; \S 20.5])$. An m-orthocomplete Boolean algebra satisfying the $m$-chain condition is complete.

\section{REFERENCES}

1. S. S. Holland, Jr., "The current interest in orthomodular lattices," in Trends in lattice theory, Van Nostrand, Princeton, N. J., 1969.

2. R. Sikorski, Boolean algebras, 2nd ed., Academic Press, New York and SpringerVerlag, Berlin, 1964. MR 31 \#2178.

3. N. Zierler, Axioms for non-relativistic quantum mechanics, Pacific J. Math. 11 (1961), 1151-1169. MR 25 \#4385.

University of Massachusetts, Amherst, Massachusetts 01003 